\title{
Human Herpes Virus 8/Epstein-Barr Virus-Copositive, Plasmablastic Microlymphoma Arising in Multicentric Castleman's Disease of an Immunocompetent Patient
}

\author{
Yong-Moon Lee · Jin-Man Kim · Sam-Yong Kim ${ }^{1}$ \\ Departments of Pathology and Medical Science and ${ }^{1}$ Internal Medicine, Chungnam National University School of Medicine, Daejeon, Korea
}

Castleman's disease is a lymphoproliferative disorder characterized by expanded germinal centers with B-cell proliferation and vascular proliferation. When infected with human herpes virus 8 (HHV8), it may develop multicentric Castleman's disease (MCD) from polyclonal isolated HHV8-positive plasmablasts to monoclonal microlymphomas and frank plasmablastic lymphomas. ${ }^{1}$ In MCD-associated plasmablastic microlymphomas, a few cases of HHV8/Epstein-Barr virus (EBV) coinfection to human immunodeficiency virus (HIV)-positive patients have been known. ${ }^{2}$ Here, we describe a plasmablastic microlymphoma arising in MCD showing HHV8/EBV co-positivity especially in HIV-seronegative patient.

\section{CASE REPORT}

A 53-year-old man was referred to Chungnam National University Hospital complaining of abdominal distention with epigastric discomfort. He denied weight loss, fever, malaise, or any systemic symptoms, and had no remarkable previous medical history. His laboratory test results were within normal ranges and anti-HIV antibody was negative. Computed tomographic scans revealed enlargement of multiple intraabdominal lymph nodes (Fig. 1A). Exploratory laparotomy was done and a huge retroperitoneal lymph node was submitted for the pathological diagnosis (Fig. 1B). The histologic examination demonstrated

Corresponding Author

Sam-Yong Kim, MD

Department of Internal Medicine, Chungnam National University School of Medicine,

266 Munhwa-ro, Jung-gu, Daejeon 35015, Korea

Tel: +82-42-280-7153, Fax: +82-42-280-7189, E-mail: frkim@cnu.ac.kr

Received: August 29, 2016 Revised: September 29, 2016

Accepted: September 30, 2016 marked lymphoid follicular hyperplasia with vascular hyalinization showing classical features of Castleman's disease (Fig. 2A, $\mathrm{B})$, and the interfollicular area/sinuses showed a dense infiltrate of large lymphoplasmablastic cells (Fig. 2C, D). These atypical cells had the characteristics of large round or oval nuclei with the irregular nuclear contours similar to plasmablasts, which replaced the periphery of the follicles making cohesive sheets of microlymphoma (Fig. 2D). The immunohistochemical studies showed that the large atypical cells were positive for MUM-1/IRF4, epithelial membrane antigen, HHV8, and EBV encoded ribonucleic acids by in situ hybridization (Fig. 3), whereas CD45, CD20, CD79a, PAX5, CD10, Bcl-6, CD4, CD5, CD8, CD138, and CD30 were negative and did not express immunoglobulin heavy or light chains. Interestingly, CD 3 was expressed aberrantly throughout the atypical cells, which can occasionally be seen in HHV8/EBV copositive lymphomas. ${ }^{3}$ Due to these findings, the plasmablastic microlymphoma arising in multicentric Castleman disease was made. The patient received cyclophosphamide, doxorubicin, vincristine and predinisolone mixed chemotherapy, and has shown to be in a stable state without disease progression for 12 months.

\section{DISCUSSION}

The MCD, initially separated from the Castleman's disease by its multicentric nature, is now considered as a different disease with different etiology; however, in some cases, the clinical outcome can be much worse. The patients may present fever, sweats, fatigue, lymphadenopathy, and splenomegaly which can be often fatal. ${ }^{4}$

The HHV8 is well known as the pathogenesis of Kaposi's sarcoma, primary effusion lymphoma (PEL), MCD, and plasma- 
blastic proliferations. Although not in localized Castleman's disease, HIV-infected cases of MCD are always accompanied with HHV8 infection, and HIV-negative cases show 40\%-50\% of HHV8 infection. ${ }^{5}$ This HHV8-associated MCD, also known as a plasmablastic variant of MCD, is characterized by the presence of plasmablasts scattered in the mantle zones of the follicles and the plasmablasts harbor HHV8 with both latent (latency associated nuclear antigen, LANA+) and lytic (viral homolog of human interleukin 6, vIL6+) profile. When scattered HHV8infected plasmablasts make confluent clusters, sometimes to form foci or large sheets, the plasmablastic microlymphoma or frank lymphoma should be considered. ${ }^{6}$
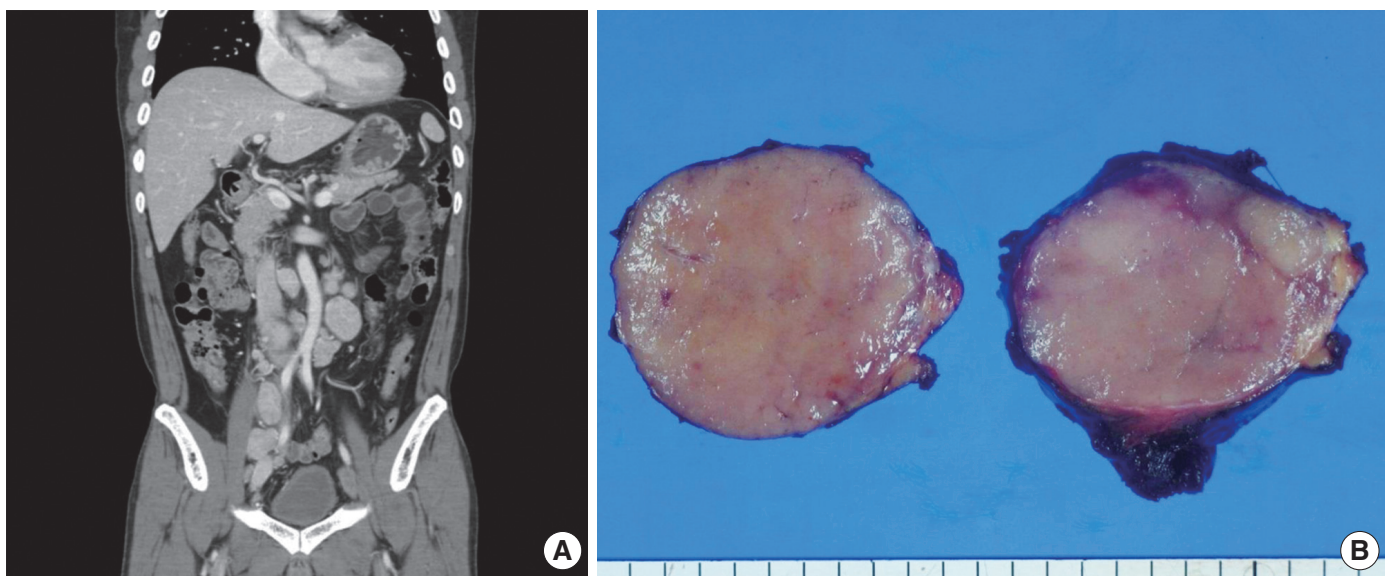

Fig. 1. Imaging and macroscopic findings of the lymph node. (A) Abdominal computed tomography showing multiple enlarged retroperitoneal lymph nodes along the axis. (B) A 5.0-cm round and tan to pinkish contexture demonstrated with vague lobulation on cut section.
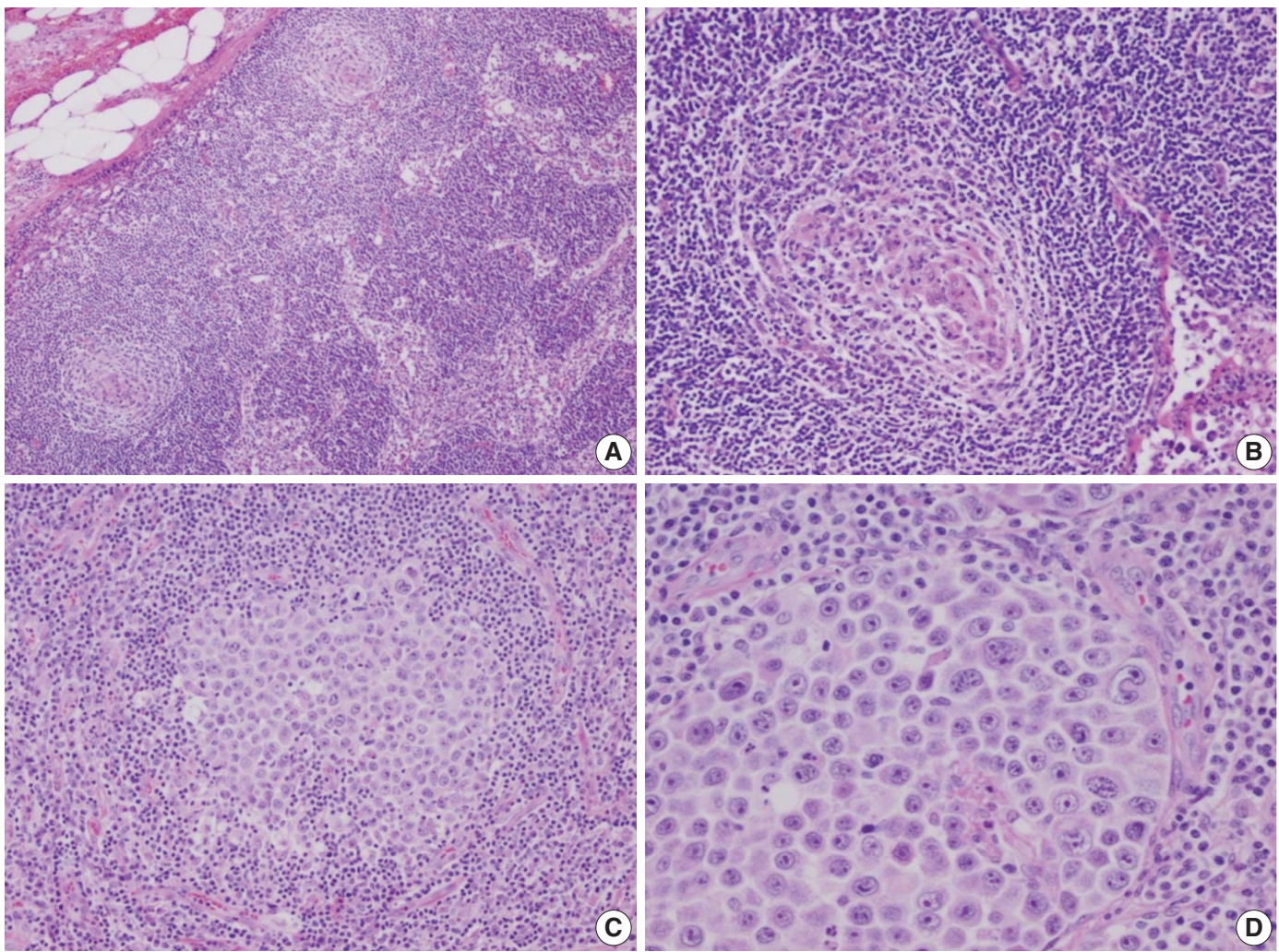

Fig. 2. Microscopic findings of the lymph node. (A) Low-magnification showing multiple, well-formed germinal centers. (B) The hyalinized vascular proliferations surrounding follicles characteristic of Castleman's disease. (C) The confluent aggregates of plasmablasts in the germinal centers indicating the germinotrophism. (D) High-magnification showing the invasion of plasmablasts to the interfollicular spaces with sinusoidal pattern. 
Certain HHV8 elements have been known to show oncogenic properties, especially vIL6, which is thought to underlie the pathogenesis of HHV8-associated MCD. When expressed constantly in mice, vIL6 can induce symptoms that resemble human MCD. These symptoms can be ameliorated by the blockade of interleukin 6 (IL-6) signaling with anti-IL-6 receptor antibodies. ${ }^{7}$ When naive $\mathrm{B}$ cells are infected by EBV, they drive proliferation and expansion of the EBV-infected B-cell pool, analogous to patterns observed in germinal centers via expressing viral latency III or nuclear antigens such as Epstein-Barr nuclear antigen 1 (EBNA) 1, 2, 3A, 3B, 3C, -LP, and latent membrane proteins (LMP1, 2A, and $2 \mathrm{~B}){ }^{8}$ Interactions among co-infecting viruses may increase cellular transformation and oncogenesis especially in lymphomagenesis which is suggested by the simultaneous detection of co-infecting viruses within the same neoplastic cells harboring HHV8 and EBV infection. In the majority of PEL, the HHV8-positive lymphoma cells are co-infected by EBV, and the analysis of EBV gene expression in PEL shows expression of EBNA1 but not LMP1. Nevertheless, the precise oncogenic mechanism of EBV in PEL remains to be investigated.'

In contrast to PEL, HHV8-associated MCD is usually associated with HIV but not with EBV infection and, only a few cases of plasmablastic microlymphoma arising in MCD showing HHV8/EBV co-positivity to HIV-positive patients have been known. Our case demonstrates obvious plasmablastic proliferations in the background of MCD. These infiltrative plasmablasts composed of confluent clusters and sheets, should be interpretated as microlymphoma positive for HHV8, and EBV in HIV-seronegative patients, has not been reported. The germinotropic lymphoproliferative disorder has histologic features similar to those of plasmablastic microlymphoma. In contrast to plasmablastic microlymphoma, it affects localized lymphadenopathy without systemic symptoms and HHV8 infected cells are confined to the germinal centers without MCD background showing a good outcome. ${ }^{10}$

In summary, we describe a case of plasmablastic microlymphoma arising in MCD showing HHV8/EBV co-positivity, and occurring in an immunocompetent patient.
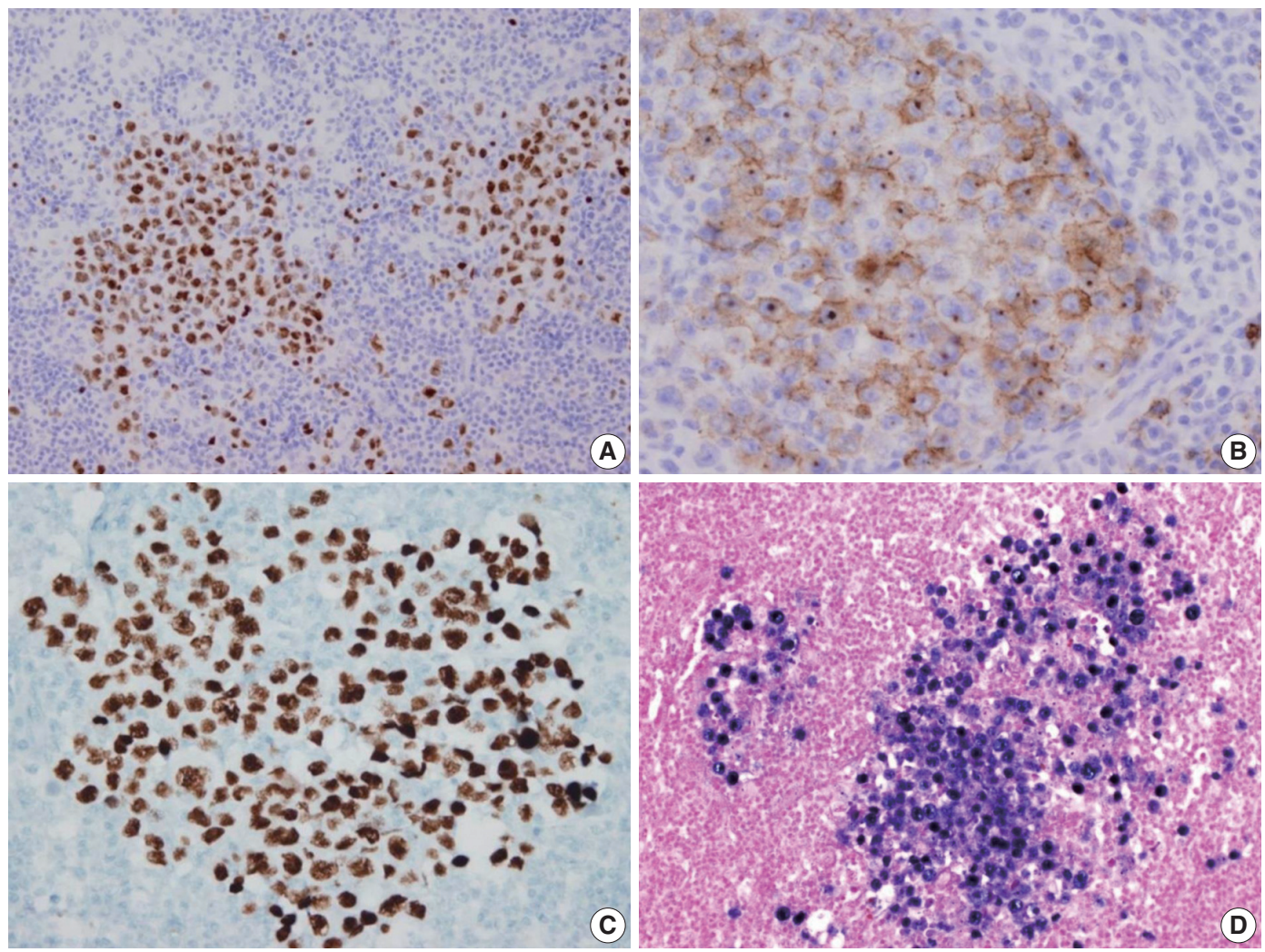

Fig. 3. Immunohistochemical staining results of the lymph node. (A) MUM-1/IRF4-stain highlighting sheets of germinotrophic plasmablasts. (B) Epithelial membrane antigen-stain demonstrating confluent aggregation of plasmablasts with sinusoidal pattern. (C) Human herpes virus 8 encoded latency associated nuclear antigen 1 highlighting the atypical plasmablasts. (D) In situ hybridization for Epstein-Barr virus (EBV) encoded ribonucleic acids showing coinfection of EBV in the atypical plasmablasts. 


\section{Conflicts of Interest}

No potential conflict of interest relevant to this article was reported.

\section{Acknowledgments}

This study was funded by research fund of Chungnam National University in 2010.

\section{REFERENCES}

1. Du MQ, Liu H, Diss TC, et al. Kaposi sarcoma-associated herpesvirus infects monotypic (IgM lambda) but polyclonal naive B cells in Castleman disease and associated lymphoproliferative disorders. Blood 2001; 97: 2130-6.

2. Seliem RM, Griffith RC, Harris NL, et al. HHV-8+, EBV+ multicentric plasmablastic microlymphoma in an HIV+ Man: the spectrum of HHV-8+ lymphoproliferative disorders expands. Am J Surg Pathol 2007; 31: 1439-45.

3. Dong HY, Wang W, Uldrick TS, Gangi M. Human herpesvirus 8and Epstein-Barr virus-associated solitary B cell lymphoma with a T cell immunophenotype. Leuk Lymphoma 2013; 54: 1560-3.
4. Wang HW, Pittaluga S, Jaffe ES. Multicentric Castleman disease: where are we now? Semin Diagn Pathol 2016; 33: 294-306.

5. Chadburn A, Cesarman E, Nador RG, Liu YF, Knowles DM. Kaposi's sarcoma-associated herpesvirus sequences in benign lymphoid proliferations not associated with human immunodeficiency virus. Cancer 1997; 80: 788-97.

6. Dupin N, Diss TL, Kellam P, et al. HHV-8 is associated with a plasmablastic variant of Castleman disease that is linked to HHV8-positive plasmablastic lymphoma. Blood 2000; 95: 1406-12.

7. Katsume A, Saito H, Yamada Y, et al. Anti-interleukin 6 (IL-6) receptor antibody suppresses Castleman's disease like symptoms emerged in IL-6 transgenic mice. Cytokine 2002; 20: 304-11.

8. Vockerodt M, Yap LF, Shannon-Lowe C, et al. The Epstein-Barr virus and the pathogenesis of lymphoma. J Pathol 2015; 235: 312-22.

9. Fassone L, Bhatia K, Gutierrez M, et al. Molecular profile of Epstein-Barr virus infection in HHV-8-positive primary effusion lymphoma. Leukemia 2000; 14: 271-7.

10. Ferry JA, Sohani AR, Longtine JA, Schwartz RA, Harris NL. HHV8-positive, EBV-positive Hodgkin lymphoma-like large B-cell lymphoma and HHV8-positive intravascular large B-cell lymphoma. Mod Pathol 2009; 22: 618-26. 